\title{
Stimulating illusory own-body perceptions
}

The part of the brain that can induce out-of-body experiences has been located.

'O ut-of-body' experiences (OBEs) are curious, usually brief sensations in which a person's consciousness seems to become detached from the body and take up a remote viewing position $^{1-3}$. Here we describe the repeated induction of this experience by focal electrical stimulation of the brain's right angular gyrus in a patient who was undergoing evaluation for epilepsy treatment. Stimulation at this site also elicited illusory transformations of the patient's arm and legs (complex somatosensory responses) and whole-body displacements (vestibular responses), indicating that out-of-body experiences may reflect a failure by the brain to integrate complex somatosensory and vestibular information ${ }^{1-3}$.

Our patient was a 43-year-old, righthanded woman who had suffered from complex partial seizures for 11 years; right temporal-lobe epilepsy was implicated. As magnetic-resonance imaging did not reveal any lesion, invasive monitoring was undertaken to localize the seizure focus precisely. Subdural electrodes were implanted to record seizures, and focal electrical stimulation was used to identify the vital cortex ${ }^{4}$.

Figure 1 shows the results of stimulation mapping and the electrode site on the right angular gyrus where stimulation repeatedly induced OBEs, as well as vestibular and complex somatosensory responses. $M$ apping of motor, somatosensory and auditory functions revealed no deviant brain pathology in this patient with respect to anatomical representations of cortical functions. The epileptic focus was located more than $5 \mathrm{~cm}$ anterior to the stimulation site, in the medial temporal lobe; electrical stimulation of this site did not induce OBEs, and these experiences were not part of the patient's habitual seizures.

Initial stimulations $(\mathrm{n}=3 ; 2.0-3.0 \mathrm{~mA})$ induced vestibular responses, in which the patient reported that she was "sinking into the bed" or "falling from a height". Increasing the current amplitude (3.5 mA) led to an OBE ("I see myself lying in bed, from above, but I only see my legs and lower trunk"). Two further stimulations induced the same sensation, which included an instantaneous feeling of "lightness" and "floating" about two metres above the bed, close to the ceiling.

The patient was then asked to watch her (real) legs during the electrical stimulation $(n=2 ; 4.0,4.5 \mathrm{~mA})$. As before, she was lying down (upper body supported at an angle of $45^{\circ}$, legs outstretched). This time, she reported seeing her legs "becoming shorter".

Figure 1 Three-dimensional surface reconstruction of the right hemisphere of the brain from magnetic-resonance imaging. Subdural electrodes were implanted in the brain of an epileptic patient undergoing presurgical evaluation; the locations at which focal electrical stimulation (ES) evoked behavioural responses are shown: magenta, motor; green, somatosensory cortex; turquoise, auditory cortex. Yellow, site at which out-ofbody experience $(\mathrm{OBE})$, bodypart illusions and vestibular responses were induced (arrow). Stars indicate the epileptic focus in the medial temporal lobe. Informed con-

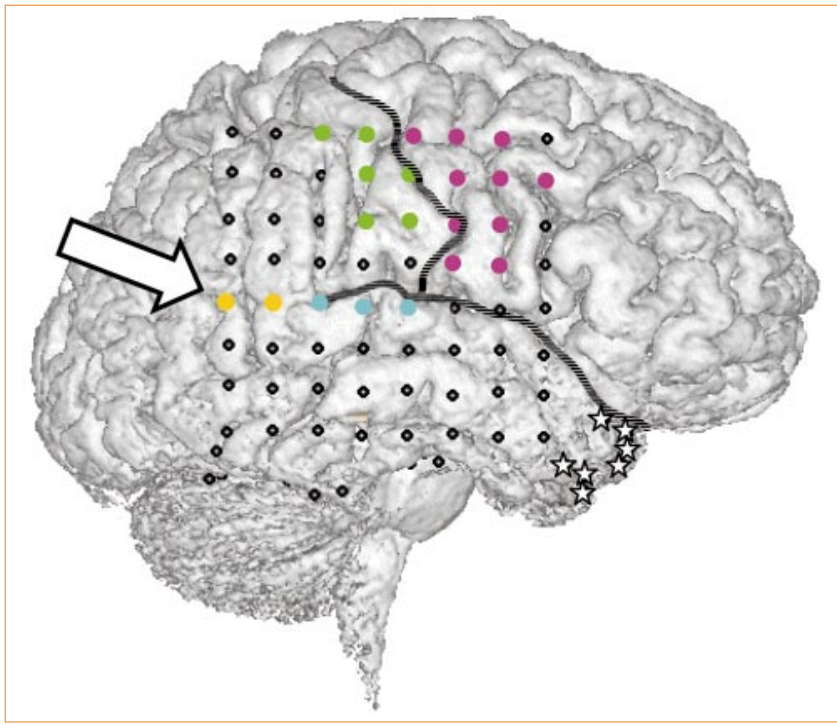
sent was obtained from the patient and ES procedures conformed to the Declaration of Helsinki. Constant current (0.5-5.0 mA, 2-s train duration) was applied at $50 \mathrm{~Hz}$ in a bipolar manner through adjacent contacts ${ }^{4}$. Since undergoing a right anterior temporal lobectomy in 2000 , the patient has been free of complex partial seizures.

If the patient's legs were bent before the stimulation ( $90^{\circ}$ knee angle; $n=2 ; 4.0,5.0$ $m A)$, she reported that her legs appeared to be moving quickly towards her face, and took evasive action.

When asked to look at her outstretched arms during the electrical stimulation $(n=2 ; 4.5,5.0 \mathrm{~mA})$, the patient felt as though her left arm was shortened; the right arm was unaffected. If both arms were in the same position but bent by $90^{\circ}$ at the elbow, she felt that her left lower arm and hand were moving towards her face $(n=2$; 4.5, 5.0 mA). When her eyes were shut, she felt that her upper body was moving towards her legs, which were stable ( $n=2$; 4.0, 5.0 mA).

These observations indicate that OBEs and complex somatosensory illusions can be artificially induced by electrical stimulation of the cortex. The association of these phenomena and their anatomical selectivity suggest that they have a common origin in body-related processing ${ }^{1-3}$, an idea that is supported by the restriction of these visual experiences to the patient's own body.

During her OBE, the patient only 'saw' that part of her body that she also felt was modified during her body-transformation experiences. This contrasts with the noncorporeal visual hallucinations that are commonly induced by electrical stimulation at the parieto-temporal junction ${ }^{5}$. As suggested by previous neurological investigations on OBEs ${ }^{1-3}$ and other body-cognition disorders $\mathrm{s}^{6-8}$, the angular gyrus could be a crucial node in a larger neural circuit that mediates complex own-body perception.

Out-of-body and body-transformation experiences are transitory and may disappear when a person attempts to inspect the illusory body or body part ${ }^{1-3}$. Our findings suggest that changes in visual attention and/or current amplitude in the angular gyrus ${ }^{4,9}$ could bring about these phenomenological modifications.

Although we do not fully understand the neurological mechanism that causes OBEs, our results imply that vestibular processing $^{2}$ may be important. Although translational vestibular responses were evoked initially without an OBE and can be produced in isolation ${ }^{4}$, vestibular sensations of levitation and lightness ${ }^{1-3}$ accompanied OBEs in our patient. Also, the core region of the human vestibular cortex is situated close to the angular gyrus ${ }^{10}$. It is possible that the experience of dissociation of self from the body is a result of failure to integrate complex somatosensory and vestibular information.

Olaf Blanke*t, Stéphanie Ortiguet, Theodor Landist, Margitta Seeck*

*Laboratory of Presurgical Epilepsy Evaluation, Program of Functional Neurology and Neurosurgery, U niversity Hospitals of Geneva and Lausanne, Geneva 1211 and Lausanne 1011, Switzerland

e-mail: olaf.blanke@hcuge.ch

†Functional Brain M apping Laboratory,

Department of Neurology, Geneva University

H ospital, 1211 Geneva, Switzerland 
1. Brugger, P, Regard, M \& Landis, T. Cogn. Neuropsychiatr. 2 19-38 (1997)

2. Grüsser, O. J. \& Landis, T. Visual Agnosias and Other Disturbances of Visual Perception and Cognition 297-303 (M acmillan, Amsterdam, 1991)

3. Hécaen, H. \& Ajuriaguerra, J. M éconnaissances et H allucinations Corporelles 310-343 (Masson, Paris, 1952).

4. Blanke, O., Perrig, S., Thut, G., Landis, T. \& Seeck, M. J. Neurol. Neurosurg. Psychiatr. 69, 553-556 (2000)

5. Penfield, W. \& Perot, P. Brain 86, 595-696 (1963)

\section{Eukaryotic evolution}

\section{Early origin of canonical introns}

pliceosomal introns, one of the hallmarks of eukaryotic genomes, were thought to have originated late in evoIution ${ }^{1,2}$ and were assumed not to exist in eukaryotes that diverged early - until the discovery of a single intron with an aberrant splice boundary in the primitive 'protozoan' Giardia”. Here we describe introns from a close relative of Giardia,

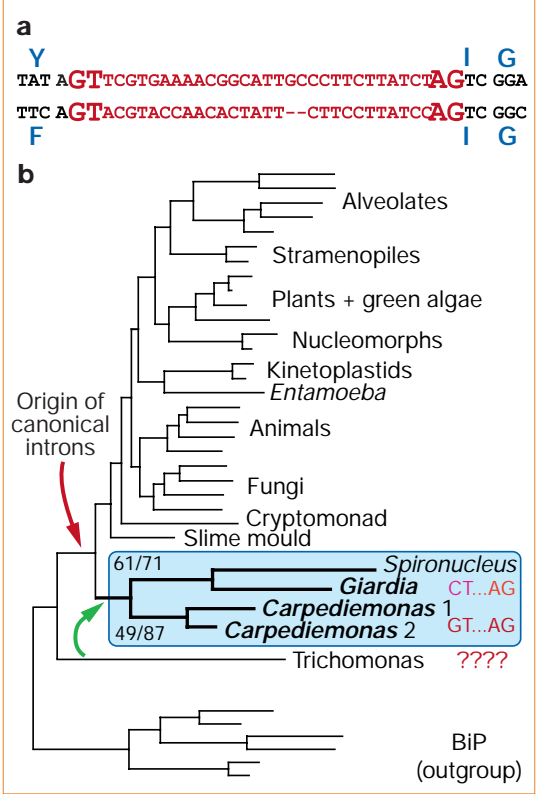

Figure 1 Introns and evolutionary affinities of Carpediemonas. a, Portions of two Carpediemonas carbamate kinase genes, showing intron sequences (red) interrupting the protein-coding sequence (in blue). The introns have canonical splice boundaries (GT... AG; large red type). b, Maximum-likelihood evolutionary tree of eukaryotic cytosolic Hsp70 proteins (' $\mathrm{T}+$ invariable sites' model). Endoplasmic-reticulum Hsp70 ('BiP') is used as an outgroup. The grouping of Carpediemonas with Giardia and Spironucleus is shown in the blue box; statistical support (bootstrap percentages) for this grouping is assessed using likelihood (upper left of box), and likelihood distance (lower left). The higher percentages (right in each pair) apply when the outgroup is omitted. The basal placement of Trichomonas is weakly supported with likelihood (21\%); green arrow shows a more plausible position on the basis of other evidence ${ }^{7,13}$. The intron splice boundaries for the relevant groups and the origin of canonical introns are shown in red. New sequences have been deposited at GenBank under accession numbers AY131204-AY131209.
6. Damasio, A. The Feeling of What $\mathrm{H}$ appens: Body, Emotions and the M aking of Consciousness 213-215 (Vintage, London, 2000). 7. Worthington, A. \& Beevers, L. Neurocase 2, 135-140 (1996).

8. Halligan, P. W., Marshall, J. C. \& Wade, D. T. Cortex 31, 173-182 (1995).

9. Nathan, S. S., Sinha, S. R., Gordon, B., Lesser, R. P. \& Thakor, N. V. Electroencephalogr. Clin. Neurophysiol. 86, 183-192 (1993). J. Neurophysiol. 80, 2699-2709 (1998)

Competing financial interests: declared none 10. Lobel, E., Kleine, J., Leroy-Wilig, A., Le Bihan, D. \& Berthoz, A.

spliceosomal introns might then be a later innovation in more modern cells. Our results indicate that this is not the case, however, as canonical introns seem to be an ancestral feature of the larger evolutionary grouping that includes Giardia and Carpediemonas. The aberrant Giardia intron probably represents a lineage-specific (or intron-specific) secondary alteration of the 5' splice boundary.

The extremely early divergence attribCarpediemonas membranifera, that have boundary sequences of the normal eukaryotic type, indicating that canonical introns are likely to have arisen very early in eukaryotic evolution.

Carpediemonas membranifera is a poorly studied, free-living microbial eukaryote that is considered to be a relative of Giardia on the basis of its morphology ${ }^{4}$. Using the polymerase chain reaction (PCR) with Carpediemonas genomic DNA as template, we determined the partial sequences of two distinct carbamate kinase genes from this organism. In both genes, an insertion of 33 or 31 nucleotides interrupts the similar protein-coding sequence shared with carbamate kinase genes from other organisms (Fig. 1a). These insertions are bounded by guanine and thymine (GT) nucleotides at the $5^{\prime}$ end and adenine and guanine (AG) nucleotides at the $3^{\prime}$ end, which is a characteristic of most of the spliceosomal introns that interrupt protein-coding genes in other eukaryotes.

We used PCR with reverse transcription to recover the messenger RNA sequence of one of the two Carpediemonas carbamate kinase genes. This sequence lacks the insertion, which is presumably removed (spliced) from the messenger RNA before translation. We conclude that the insertions in the Carpediemonas carbamate kinase genes are canonical 'GT... AG' spliceosomal introns, al beit comparatively small ones.

To determine the evolutionary affinities of Carpediemonas, we used PCR to amplify near-complete sequences for two genes that encode cytosolic heat-shock protein 70 (H sp 70). We also sequenced a cloned H sp70 gene from Spironucleus barkhanus, a very close relative of Giardia. Maximum likelihood analysis of $\mathrm{Hsp} 70$ proteins reveals a specific evolutionary relation between Carpediemonas, Giardia and Spironucleus (Fig. 1b); three other molecular markers also support this relationship ${ }^{5}$.

The single intron found in a Giardia gene has a non-canonical CT dinucleotide at its $5^{\prime}$ splicing boundary ${ }^{3}$, which could be interpreted as a 'frozen' primitive eukaryotic condition: canonical 'GT ... AG' uted to Giardia is based on the absence or aberration of many typical eukaryotic features, such as mitochondria and introns, and on its arguably deep-branching position in many phylogenetic trees ${ }^{6-9}$. The grouping of Giardia with Carpediemonas (which, as well as canonical introns, has organelles that are probably derived from mitochondria ${ }^{4}$ ) weakens this argument for early divergence.

Irrespective of the true evolutionary position of Giardia, the only potentially 'early' eukaryotic group in which introns have not been found are the parabasalids, such as Trichomonas ${ }^{10,11}$. Trichomonas is already known to possess some of the cellular machinery for intron splicing ${ }^{12}$, howis evolutionarily affiliated with Giardia and its relatives ${ }^{7,13}$ (and is slightly misplaced in many phylogenies, including that shown in Fig. 1b). An affiliation with Giardia implies a similar closeness to Carpediemonas, and it is likely that parabasalids have, or had, canonical introns. There is now every were present in the most recent common ancestor of living eukaryotes.

Alastair G. B. Simpson, Erin K. MacQ uarrie, Andrew J. Roger

Canadian Institute for Advanced Research, Program in Evolutionary Biology, Department of Biochemistry and M olecular Biology, Dalhousie University, Halifax, Nova Scotia B3H 4H 7, Canada e-mail: simpson@hades.biochem.dal.ca

1. Palmer, J. D. \& Logsdon, J. M. Curr. O pin. Genet. Dev. 1, 470-477 (1991)

2. Logsdon, J. M. Curr. Opin. Genet. Dev. 8, 637-648 (1998).

3. Nixon, J. E. J. et al. Proc. Natl Acad. Sci. USA 99, 3701-3705 (2002).

4. Simpson, A. G. B. \& Patterson, D. J. Eur. J. Protistol. 35, 353-370 (1999).

5. Simpson, A. G. B. et al. M ol. Biol. Evol. 19, 1782-1791 (2002).

6. Cavalier-Smith, T. Trends Genet. 7, 145-148 (1991).

7. Embley, T. M . \& Hirt, R. P. Curr. O pin. Genet. Dev. 8, 624-629 (1998)

8. Roger, A. J. Am. Nat. 154 (suppl.), 146-163 (1999).

9. Sogin, M. L. Curr. O pin. Genet. Dev. 7, 792-799 (1997)

10. Johnson, P. J. Proc. N atl Acad. Sci. USA 99, 3359-3361 (2002).

11. Archibald, J. M., O'Kelly, C. J. \& Doolittle, W. F. Mol. Biol. Evol. 19, 422-431 (2002).

12. Fast, N. M., Logsdon, J. M. \& Doolittle, W. F. M ol. Biochem. Parasitol. 99, 514-522 (1999)

13.Dacks, J. B. \& Roger, A. J.J. Mol. Evol. 48, 779-783 (1999). Competing financial interests: declared none. ever, and there is evidence to indicate that it reason to assume that canonical introns

brief communications is intended to provide a forum for both brief, topical reports of general scientific interest and technical discussion of recently published material of particular interest to non-specialist readers. Priority will be given to contributions that have fewer than 500 words, 10 references and only one figure. Detailed guidelines are available on Nature's website (www.nature.com) or on request from nature@ nature.com 